\title{
PRIESTLEY SPACES
}

\author{
P. VENUGOPALAN
}

(Communicated by Louis J. Ratliff, Jr.)

\begin{abstract}
We give a purely order-theoretic characterization of complete lattices that are compact totally order-disconnected (Priestley) spaces with respect to the Lawson topology. We also characterize complete lattices that are Priestley spaces with respect to the interval topology.
\end{abstract}

\section{INTRODUCTION}

Let $P$ be a partially ordered set with a topology defined on it. $P$ is called totally order-disconnected if for $x, y \in P$ with $x \not y y$, there exists a closedopen upper set containing $x$ but not $y$. The interest in these spaces is mainly due to the celebrated Priestley Duality. H. A. Priestley proved that the category of bounded distributive lattices and 0,1-preserving lattice homomorphisms and the category of compact totally order-disconnected spaces (Priestley spaces) and order-preserving continuous maps are dually equivalent. See [5] and [6]. Also see [7] for an excellent survey of this and related dualities. Hochster [4] showed that a partially ordered set is a Priestley space if and only if it is isomorphic to the set of prime ideals, ordered by inclusion, of a commutative ring with identity. But an order-theoretic characterization of partially ordered sets, which can be topologized to create Priestley spaces, is still lacking. In this paper we give a purely order-theoretic characterization of complete lattices that are Priestley spaces with respect to the Lawson topology. We also characterize complete lattices that are Priestley spaces with respect to the interval topology.

Let $P$ be a complete lattice and let $A \subseteq P$. The set $\{x: x \geq a$ for some $a \in A\}$ is denoted by $\uparrow A$, and the set $\{x: x \leq a$ for some $a \in A\}$ is denoted by $\downarrow A$. The topology, generated by $\downarrow x, x \in P$ as subbasic closed sets, is called the upper topology. The lattice of upper-open sets, under inclusion, is denoted by $\nu(P)$. The topology, generated by $\uparrow x, x \in P$ as subbasic closed sets, is called the lower topology. The lattice of lower-open sets is denoted by $\omega(P)$. The interval topology is the supremum of the lower and the upper topologies. A subset $U$ of $P$ is called Scott-open if (i) $U=\uparrow U$, and (ii) if $D$ is a directed

Received by the editors June 15, 1989 and, in revised form, September 11, 1989.

1980 Mathematics Subject Classification (1985 Revision). Primary 06B35, 06B30, 54F05.

Key words and phrases. Complete lattice, Priestley space, Lawson topology, interval topology. 
subset of $P$ with $\sup D \in U$, then $U \cap D \neq \varnothing$. It is easily verified that Scottopen sets form a topology, which is called the Scott topology, and is denoted by $\sigma(P)$. The Lawson topology is the common refinement of the lower and the Scott topologies.

\section{The LAwSON TOPOLOGY}

In this section we give a characterization of complete lattices that are Priestley spaces when endowed with the Lawson topology. The key ingredient is the generalized way-below relation introduced by Gierz, Lawson, and Stralka [3]. For subsets $A$ and $B$ of a partially ordered set $P$, we say $A$ is way-below $B$, written $A \ll B$, if whenever $D$ is a directed subset of $P$ for which $\sup D$ exists and is in $\uparrow B$, then $D \cap \uparrow A \neq \varnothing$. Recall that a complete lattice is called algebraic if every element is a sup of compact elements ( $x$ is called compact if $x \ll x)$.

1.1. Definition. A complete lattice $P$ is called pseudoalgebraic if for each $x \in$ $P, \uparrow x=\cap\{\uparrow A: A \ll A, A$ is finite, and $x \in \uparrow A\}$.

Let $P$ be the set of all nonnegative integers. Let $L=P \cup\{a, b\}$. In $L, x \leq y$ if and only if (i) $x \leq y$ in $P$, (ii) $x=0$, or (iii) $y=b$. It is easy to verify that $L$ is a pseudoalgebraic lattice. But $L$ is not algebraic; $a$ is not the sup of compact elements below it.

Notice that in any complete lattice, if $A \ll x$, and $B \ll x$, then there exists a $C \ll x$ such that $C \subseteq \uparrow A \cap \uparrow B$. For example, $C=\{x \vee y: x \in A$ and $y \in B\}$. Therefore the set $\{\uparrow A: A \ll A, x \in \uparrow A, A$ is finite $\}$ is down directed.

In [2] a complete lattice $L$ is called a generalized continuous lattice (GCL, for short) if for all $x \in L, \uparrow x=\cap\{\uparrow A: A$ is finite, $A \ll x\}$. There it was shown that a complete lattice has a Hausdorff Lawson topology if and only if it is a GCL. In a GCL if $U$ is Scott-open with $x \in U$, then there exists a finite set $F \subseteq U$ such that $x \in\{y: F \ll y\} \subseteq U$. Also see [3].

1.2. Theorem. A complete lattice is a Priestley space with respect to the Lawson topology if and only if it is pseudoalgebraic.

Proof. Let $L$ be a pseudoalgebraic lattice. Suppose $x \not y$. Since $L$ is pseudoalgebraic, there exists a finite set $F \subseteq L$ such that $F \ll F, x \in \uparrow F$, and $y \notin \uparrow F$. Clearly $\uparrow F$ is open-closed in the Lawson topology. Since the Lawson topology is compact in all complete lattices, this completes the proof that $L$ is a Priestley space.

Now suppose $L$ is a Priestley space with respect to the Lawson topology. Let $x \nless y$. Then there exists a closed-open upper set $U$ such that $x \in U$, and $y \notin U$. Since Lawson-open upper sets are Scott-open, $U$ is Scott-open and Scott-quasicompact. But the Hausdorffness of the Lawson topology implies that

$$
U=\bigcup_{\text {finite } F \subseteq U}\{y: F \ll y\} .
$$


By the quasicompactness of $U$,

$$
U=\bigcup_{i=1}^{n}\left\{y: F_{i} \ll y\right\} .
$$

Thus

$$
U=\uparrow F, \quad \text { where } F=\bigcup_{i=1}^{n} F_{i} .
$$

Clearly, since $\uparrow F$ is Scott-open, $F \ll F$. This completes the proof of the theorem.

Now we shall characterize pseudoalgebraic lattices in terms of the lower topology and the Scott topology.

1.3. Lemma. Let $L$ be a complete lattice and let $U \in \omega(L)$, the lattice of lower-open sets. Then $U \ll U$ if and only if $U$ is Scott-closed.

Proof. Suppose $U$ is Scott-closed. Let $U \subseteq \bigcup\{L \backslash \uparrow x: x \in A\}=L \backslash \uparrow y$ where $y=\sup A$. Then $\uparrow y \subseteq L \backslash U$. Since $L \backslash U$ is Scott-open, there exists a finite set $F \subseteq A$ such that $\sup F \in L \backslash U$. That is, $U \subseteq L \backslash \uparrow F=\bigcup\{L \backslash \uparrow x: x \in F\}$.

Now suppose $U \ll U$. Let $D$ be a directed set such that $D \subseteq U$. If $\sup D \notin U$, then

$$
U \subseteq L \backslash \uparrow \sup D=\bigcup_{d \in D}\{L \backslash \uparrow d\}
$$

Therefore, since $U \ll U$,

$$
U \subseteq \bigcup_{d \in G}\{L \backslash \uparrow d\}=L \backslash \uparrow \sup G
$$

for some finite $G \subseteq D$. That is, $\sup G \notin U$. This contradiction proves the lemma.

1.4. Proposition. For a complete lattice $L$ the following statements are equivalent.

(1) $L$ is a pseudoalgebraic lattice.

(2) $\omega(L)$ is an algebraic lattice.

(3) For each $\omega(L)$-closed set $C$ and $y \notin C$, there exists a finite set $F \subseteq L$ such that $F \ll F, y \notin \uparrow F$, and $C \subseteq \uparrow F$.

Proof. (1) implies (2). Suppose $A$ is a finite subset of $L$, and suppose $y \in$ $L \backslash \uparrow A$. Let $A=\left\{a_{1}, a_{2}, \cdots, a_{n}\right\}$. Then $a_{i} \not y$ for $i=1,2, \cdots, n$. Since $L$ is a pseudoalgebraic lattice, $\exists F_{i} \ll F_{i}$ such that $y \notin \uparrow F_{i}$ and $a_{i} \in \uparrow F_{i}$. Let

$$
F=\bigcup_{i=1}^{n} F_{i}
$$

Then $y \notin \uparrow F, F \ll F$ and $\uparrow A \subseteq \uparrow F$. That is, $y \in L \backslash \uparrow F \subseteq L \backslash \uparrow A$. Since $\uparrow F$ is Scott-open, $L \backslash \uparrow F$ is Scott-closed and hence, by Lemma 1.3, $L \backslash \uparrow F \ll L \backslash \uparrow F$. 
(2) implies (3). Let $C$ be $\omega(L)$-closed such that $y \notin C$. If $\omega(L)$ is an algebraic lattice, then there exists a $\omega(L)$-neighborhood $L \backslash \uparrow F \ll L \backslash \uparrow F$ such

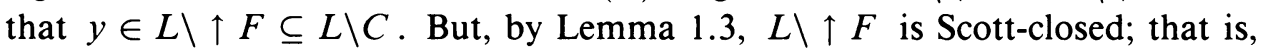
$\uparrow F$ is Scott-open. Clearly, $F \ll F$.

(3) implies (1). Let $x \nless y$. Then, by (3), there exists a finite set $F \subseteq L$ such that $F \ll F, y \notin \uparrow F$, and $\uparrow x \subseteq \uparrow F$. This shows that $L$ is pseudoalgebraic.

The following theorem is due to M. E. Rudin, a proof of which can be found in [3].

1.5. Theorem (M. E. Rudin). Let $P$ be a partially ordered set and let $F$ in $P=$ $\{\uparrow A: A$ is a finite subset of $P\} ;$ gen $:$ in $P \rightarrow 2^{P}$ is the function that assigns to each member of $F$ in $P$ the set of its minimal elements. Let $E$ be an upper set of $P$. Assume that $\Gamma$ is a descending family in $F$ in $P$ such that $\cap \Gamma \subseteq E$. Then there exists a subset $K$ of $\cup\{\operatorname{gen}(G): G \in \Gamma\}$ such that (i) $K \cap \operatorname{gen}(G) \neq \Phi$ for all $G \in \Gamma$, (ii) $K$ is a directed set, (iii) $\{\uparrow k: k \in K\}$ is a descending family with $\cap\{\uparrow k: k \in K\} \subseteq E$, and (iv) for all $F, G \in \Gamma, F \subseteq G$ implies $\operatorname{gen}(F) \cap K \subseteq \uparrow(\operatorname{gen}(G) \cap K)$.

1.6. Lemma. Let $P$ be a pseudoalgebraic lattice and let $U$ be a Scott-open set of $P$. If $x \in U$, then there exists a finite set $F \subseteq U$ such that $F \ll F$ and $x \in\{y: F \ll y\} \subseteq U$.

Proof. Suppose for all finite $F \ll F$ with $x \in \uparrow F, F \nsubseteq U$. Let $\Gamma=$ $\{\uparrow(F \backslash U): F \ll F, F$ is finite $\}$. Since $F \backslash U$ is nonempty for all $F$ and $\cap \Gamma \subseteq$ $\uparrow x$, by Rudin's Theorem, there exists a directed set $D \subseteq \bigcup\{F \backslash U: F \ll F, F$ is finite $\}$ such that $\sup D \geq x$. Since $U$ is Scott-open, there exists $d \in D$ such that $d \in U$. But then $d \in F \backslash U$ for some $F$, a contradiction. Therefore there exists a finite $F \ll F$ such that $F \subseteq U$ and $x \in \uparrow F$. Thus $x \in\{y: F \ll y\} \subseteq$ $\uparrow F \subseteq U$.

Before we state the next proposition we need to recall the following definition from [2]. A complete lattice is called hypercontinuous, if it is the image of a completely distributive lattice under a morphism preserving arbitrary infs and directed sups. We refer the reader to [2] for more details and several different characterizations of these lattices.

1.7. Proposition. For a complete lattice $L$ the following statements are equivalent.

(1) The Lawson topology on $L$ is Hausdorff, and the Scott topology has a basis of sets of the form $\uparrow F$, where $F \ll F$, and $F$ is finite.

(2) $L$ is a pseudoalgebraic lattice.

(3) $L$ is a $G C L$, and $\sigma(L)$ is an algebraic lattice.

(4) $\sigma(L)$ is hypercontinuous and algebraic.

Proof. In [2] it was shown that a complete lattice is a GCL if and only if $\sigma(L)$ is hypercontinuous. (3) if and only if (4) follows immediately from this. 
(1) implies (2). Since the Lawson topology is Hausdorff, $L$ is a GCL. Therefore if $x \not y$ there exists a Scott-open set $U$ containing $x$ such that its $\omega(L)$ closure does not contain $y[1]$. Then by (1) there exists a finite set $F \ll F$ such that $x \in \uparrow F \subseteq U$. Clearly $y \notin \uparrow F$. This shows that $L$ is pseudoalgebraic.

(2) implies (1). This follows from Lemma 1.6.

(1) implies (3). $\sigma(L)$ is algebraic since $\uparrow F$ is a compact open set for all finite sets $F \ll F$, and the Lawson topology is Hausdorff precisely when $L$ is a GCL.

(3) implies (1). Let $V$ be Scott-open and let $x \in V$. Then there exists a quasicompact open set $C$ such that $X \in C \subseteq V$. Since $L$ is a GCL,

$$
C=\bigcup_{\text {finite } F \subseteq C}\{y: F \ll y\} .
$$

Since $C$ is quasicompact,

$$
C \subseteq \bigcup_{i=1}^{n} \uparrow F_{i}=\uparrow F, \quad \text { where } F=\bigcup_{i=1}^{n} F_{i} .
$$

That is, $x \in \uparrow F=C \subseteq V$. Since $\uparrow F$ is Scott-open, $F \ll F$.

It would be interesting to investigate which results on algebraic lattices can be extended to pseudoalgebraic lattices. It can be shown that pseudoalgebraic lattices are preserved under directed-sup-preserving closure operators. See [10] for a similar result. Also every pseudoalgebraic lattice is weakly atomic; indeed, every Priestley space is weakly atomic [7].

\section{THE INTERVAL TOPOLOGY}

In this section we characterize those complete lattices that are Priestley spaces with respect to the interval topology. The following remarkable theorem due to Gierz and Lawson [2] plays a crucial role.

2.1. Theorem. Let $L$ be a complete lattice. Then the following statements are equivalent.

(1) The interval topology on $L$ is Hausdorff.

(2) $L$ is a generalized bicontinuous lattice (that is, $L$ and $L^{o p}$ are both $G C L$ and the Lawson topologies on $L$ and $L^{o p}$ agree).

In each of these cases, the interval topology and the Lawson topology agree.

We call a complete lattice $L$ pseudoalgebraic if $L$ and $L^{o p}$ are both pseudoalgebraic and the Lawson topologies on $L$ and $L^{o p}$ agree.

2.2. Theorem. For a complete lattice $L$ the following statements are equivalent.

(1) $L$ is a Priestley space with respect to the interval topology.

(2) $L$ is pseudoalgebraic.

Proof. (1) implies (2). Since the interval topology on $L$ is Hausdorff, by Theorem 2.1 , the interval topology agrees with the Lawson topologies on $L$ and $L^{o p}$. Therefore, by Theorem 1.2, $L$ is pseudoalgebraic. 
(2) implies (1). If $L$ is pseudoalgebraic, then clearly $L$ is generalized bicontinuous. Therefore, by Theorem 2.1, the interval topology is Hausdorff, and the interval topology coincides with the Lawson topologies on $L$ and $L^{o p}$. Then, by Theorem 1.2, $L$ is a Priestley space with respect to the interval topology.

It is well known that if the interval topology is Hausdorff, then that is the only topology that could make the partially ordered set a Priestley space. Thus a complete lattice in which the interval topology is Hausdorff can be made into a Priestley space if and only if it is pseudoalgebraic.

\section{REFERENCES}

1. G. Gierz, K. H. Hofmann, K. Keimel, J. D. Lawson, M. Mislove, and D. S. Scott, $A$ compendium of continuous lattices, Springer-Verlag, Berlin, Heidelberg, and New York, 1980.

2. G. Gierz and J. D. Lawson, Generalized continuous and hypercontinuous lattices, Rocky Moun tain J. Math. (1981), 271-296.

3. G. Gierz, J. D. Lawson, and A. Stralka, Quasicontinuous posets, Houston J. Math. 9 (1983), 191-208.

4. M. Hochster, Prime ideal structure in commutative rings, Trans. Amer. Math. Soc. 142 (1969), 43-60.

5. H. A. Priestley, Representation of distributive lattices by means of ordered Stone spaces, Bull. London Math. Soc. 2 (1970), 186-190.

6. Ordered topological spaces and the representation of distributive lattices, Proc. London Math. Soc. 24 (1972), 507-530.

7. Math., North-Holland, Amsterdam, 1984, pp. 39-60.

8. H. A. Priestley, Algebraic lattices as duals of distributive lattices, Lecture Notes in Pure and Applied Math., vol. 101, 1985, pp. 237-249.

9. A. R. Stralka, A partially ordered space which is not a Priestley space, Semigroup Forum 20 (1980), 293-297.

10. P. Venugopalan, Quasicontinuous posets, Semigroup Forum (to appear).

11. _ A generalization of completely distributive lattices, Algebra Universalis (to appear).

Department of Mathematics, University of Connecticut, Stamford, Connecticut 06903 